Supporting Information

\title{
Sample Size-comparable Spectral Library Enhances Data-Independent Acquisition-based Proteome Coverage of Low-input Cells
}

Asad Ali Siyal ${ }^{1,2,3 \#, ~ E r i c ~ S h e n g-W e n ~ C h e n ~}{ }^{1,5 \#}$, Hsin-Ju Chan ${ }^{1,4}$, Reta Birhanu Kitata ${ }^{1}$, Jhih-Ci Yang ${ }^{1,6,7}$, Hsiung-Lin $\mathrm{Tu}^{1,2}$ and Yu-Ju Chen ${ }^{1,2,4,6 *}$

1. Institute of Chemistry, Academia Sinica, Taipei 115, Taiwan

2. Chemical Biology and Molecular Biophysics Program, Taiwan International Graduate Program, Academia Sinica, Taipei 115, Taiwan

3. Department of Chemistry, National Tsing Hua University, Hsinchu 300, Taiwan

4. Department of Chemistry, National Taiwan University, Taipei 106, Taiwan

5. Research Center for Cancer Biology, China Medical University, Taichung 40402, Taiwan

6. Sustainable Chemical Science and Technology, Taiwan International Graduate Program, Academia Sinica and National Yang Ming Chiao Tung University, Taipei, 11529, Taiwan

7. Department of Applied Chemistry, National Yang Ming Chiao Tung University, Hsinchu 30010, Taiwan

\# co-first author

*corresponding author:

Yu-Ju Chen: yujuchen@gate.sinica.edu.tw

Institute of Chemistry, Academia Sinica, Taiwan.

Keywords: Proteomics, Data-independent Acquisition, Microproteomics, Nanoproteomics, Mass spectrometry 


\section{Contents}

Materials and Methods (Detailed).

Figure S1. Optimization of different liquid chromatography gradient lengths in DIA-MS.

Figure S2. Effect of different sizes of DDA-specific spectra libraries on the performance of protein identification in 5 cells.

Figure S3. Effect of different sizes of spectra libraries on the performance of protein identification in low-input cells by library-based DIA using DIA-NN.

Figure S4. Effect of different sizes of spectra libraries on the performance of protein and peptide identification in low-input cells by library-based DIA obtained by Orbitrap Lumos mass spectrometer using Spectronaut.

Figure S5. Evaluation of reproducibility in triplicate DIA-MS analysis identified by sample sizecomparable spectral libraries.

Figure S6. Evaluation on the spectral similarities of commonly and uniquely identified proteins in different libraries.

Figure S7. Validation of selected peptides identified in the 5-cells of PC-9 digests by parallel reaction monitoring (PRM) MS.

Table S1. Detailed Parameters of DIA-MS2 optimization for low-input cells.

Table S2. Summary of proteome coverages of the three DIA-specific spectral libraries.

Table S3. Summary of proteome coverages of the three DDA-specific spectral libraries. 


\section{Materials and Methods (Detailed).}

\section{Materials and reagents}

Tris (2-carboxyethyl) phosphine hydrochloride (TCEP), chloroacetamide (CAA), triethylammonium bicarbonate (TEABC), trifluoroacetic acid (TFA) and Formic Acid (FA) were purchased from Sigma-Aldrich (St. Louis, MO, USA). RapiGest SF surfactant was purchased from Waters (MA, USA). LC-MS/MS grade Trypsin and Lysine-C were purchased from Promega (WI, USA). BCA protein assay kit was purchased from Pierce (IL, USA). The iRT peptide kit was purchased from Biognosys AG (Schlieren-Zurich, Switzerland). Deionized water was obtained from Milli-Q Ultrapure Water Purification System (Millipore, MA, USA). The C18 Ziptips were purchased from Milipore (Millipore corporation, MA, USA).

\section{Cell culture}

The human lung adenocarcinoma cell line (PC-9) was grown in RPMI-1640 medium containing fetal bovine serum (FBS) (10\% v/v), $1 \mathrm{mM}$ sodium pyruvate, sodium bicarbonate $(2 \% \mathrm{w} / \mathrm{v}), 100$ units $/ \mathrm{ml}$ penicillin, and $100 \mu \mathrm{g} / \mathrm{ml}$ streptomycin at $37{ }^{\circ} \mathrm{C}$ in a humidified atmosphere of $5 \% \mathrm{CO} 2$ incubator.

\section{Cell lysis and proteomic sample preparation}

Cells were collected with a concentration of $5 \times 10^{5} / \mathrm{ml}$ and washed with phosphate buffer saline (PBS) three times. After washing and resuspending the cells with PBS, $10^{6}$ cells were poured into vials, centrifuged at $1000 \mathrm{rpm}$ for $3 \mathrm{~min}$, and pallet was collected in cocktail lysis buffer $(0.3 \%$ Rapigest, 10mM TCEP, 40mM CAA, protease inhibitors) to achieve one-pot lysis, reduction and alkylation. The collected cells were lysed by heating at $70^{\circ} \mathrm{C}$ for $30 \mathrm{~min}$ and sonicated at $4{ }^{\circ} \mathrm{C}$ for $5 \mathrm{~min}$. The lysate was then centrifuged at $16,000 \times \mathrm{g}$ for $20 \mathrm{~min}$ at $4{ }^{\circ} \mathrm{C}$. The supernatant was collected and BCA assay was performed to measure the protein concentration using a BCA protein assay kit. An $\sim 80 \mu \mathrm{g}$ protein lysate was digested for $16 \mathrm{~h}$ with Lys-C (protein/Lys-C 20:1 $\mathrm{w} / \mathrm{w}$ ) and trypsin (protein/trypsin 10:1 w/w) at $37^{\circ} \mathrm{C}$. The digested sample was acidified with formic acid (FA) to a final concentration of ( $5 \% \mathrm{v} / \mathrm{v}$ ) by incubating for $1 \mathrm{~h}$ at $37^{\circ} \mathrm{C}$. The resultant peptides were dried and followed by peptide desalting through the C18 Ziptip. The desalted peptides concentration was measured using a bicinchoninic acid assay (BCA) protein assay kit and measuring the absorbance of the peptides on a UV-spectrophotometer at $562 \mathrm{~nm}$ wavelength. Finally, the peptides were serially diluted to a desired concentration and subsequent small aliquots corresponding to $0.75 \mathrm{ng}$ ( $\sim 5$ cells), $1.5 \mathrm{ng}$ ( $\sim 10$ cells) and $7.5 \mathrm{ng}$ ( $\sim 50$ cells) were injected and analyzed by LC-MS/MS using the Orbitrap Eclipse mass spectrometer in triplicates, respectively. The cell count estimation is based on the estimation reported in the literature that a typical mammalian cell roughly contains $150-200$ pg of protein content per cell ${ }^{8}$.

\section{DDA LC-MS/MS analysis}


The MS instrument was operated in the positive ion mode with spray voltage set to $1.75 \mathrm{kV}, \mathrm{RF}$ lens level set at $30 \%$, and ion transfer tube heated at $305^{\circ} \mathrm{C}$ for the DDA data acquisition. Top $\mathrm{N}$ multiply charged precursors were automatically isolated and fragmented according to their intensities within the cycle time of $3 \mathrm{sec}$. Intensity threshold was set to 5E4 and 8E3 for bulk (1 ug) and low-input (15 ng and $1.5 \mathrm{ng}$ ) of PC9 digests, respectively. Full MS was scanned at a resolution of 120000 with an automatic gain control (AGC) target of 4E5 and 1E6 for bulk and low-input, respectively. Max injection time was set to $50 \mathrm{~ms}$. Mass range was set to 375-1500 $\mathrm{m} / \mathrm{z}$ and isolation width for MS/MS analysis was set to $1.4 \mathrm{~m} / \mathrm{z}$ with advanced peak determination. Normalized collision energy of high-energy collision dissociation (HCD) was set to $30 \%$. MS/MS was scanned in orbitrap at the resolution of 15000 and 60000 for bulk and lowinput, respectively. AGC target for bulk was set to $5 E 4$ with a max injection time of $22 \mathrm{~ms}$, while AGC target for low-input was set to $1.25 \mathrm{E} 5$ with a max injection time of $118 \mathrm{~ms}$.

\section{Data analysis (Spectronaut)}

The DIA raw files were analyzed using home-built project-specific spectra libraries (libDIA) as well as in library-free mode (dirDIA) using Spectronaut software ${ }^{23}$ (version 14.10.201222.47784) in stringent settings. For both the libDIA and dirDIA, the protein identification was performed against the UniProt human proteome database (Homo Sapiens: 20,386 entries, downloaded on 2021/09/23) including the iRT peptide sequences using Pulsar search engine in Spectronaut ${ }^{23}$ software filtering at $1 \%$ FDR PSM and protein level. For the protein quantitation, peak area at MS2 level was calculated using top 3 peptides per protein and minor peptide grouping was adapted based on striped sequence. The carbamidomethylation of cysteine was set as a static modification, whereas the acetylation on protein $\mathrm{N}$-terminus and oxidation on methionine were selected as variable modifications.

\section{Data analysis (DIA-NN)}

For library construction in DIA-NN (1.8), raw data were uploaded to DIA-NN, searching against the updated fasta (2021) using the following options: a maximum of two missed cleavages, a maximum of 1 variable modification, enabled $\mathrm{N}$-terminal methionine exclusion, cysteine carbamidomethylation as a fixed modification, methionine oxidation as a variable modification, a minimal allowed peptide length of 7 and maximal of 30, enabled smart-profiling and filter identification results at $1 \%$ false discovery rate. For data analysis in DIA-NN (1.8), raw data were uploaded to the DIA-NN, searching against the spectral libraries constructed using DIA-NN and the UniProt human fasta (2021) using the identical parameters with that used in the library construction.

\section{Data analysis (PRM-MS)}


For parallel reaction monitoring (PRM) validation of commonly and uniquely identified peptides, the PRM raw files were imported into Skyline 64-bit version 21.1.0.278, using the $1.5 \mathrm{ng}$ library described previously (in Spectral library construction). Each peptide was subjected to a 2-min time window, using the retention time in DIA files as a reference. Peptides were defined as identified if more than 4 transitions were detected. Peak shapes, co-elution profiles, and signalto-noise ratios were also considered. Finally, the similarity of fragment relative intensities between PRM spectra and the library was evaluated by dot product scores to ensure the identification confidence.

\section{Data availability}

All mass spectrometry raw datasets, spectral libraries, and Spectronaut quantification outputs in this study were deposited in JPOST $^{24}$ and ProteomeXchange. The accession numbers are JPST001233 for JPOST and PXD027679 for ProteomeXchange and the data can be accessed through https://repository.jpostdb.org/preview/19453011636187dbd5b8943 with access key 2609. 
(a)
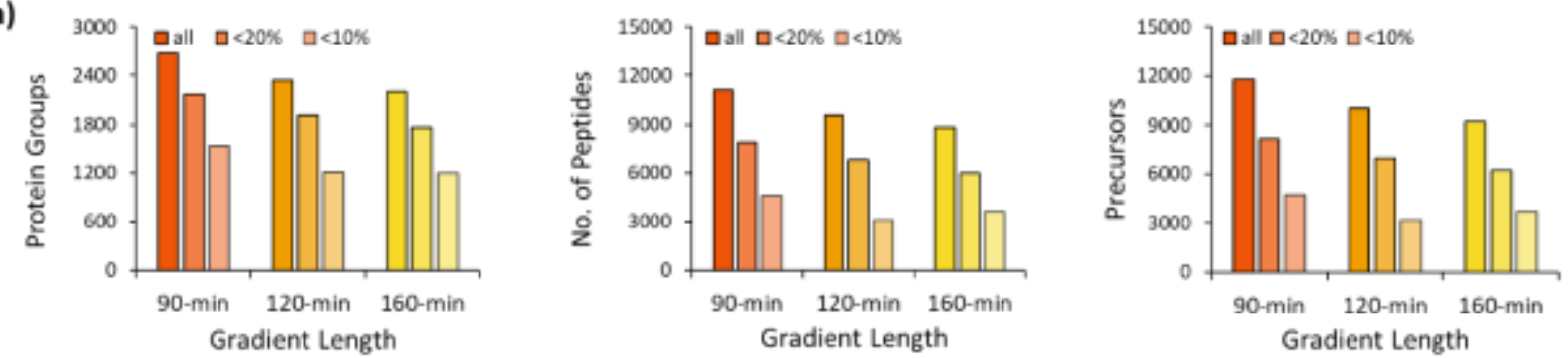

(b)

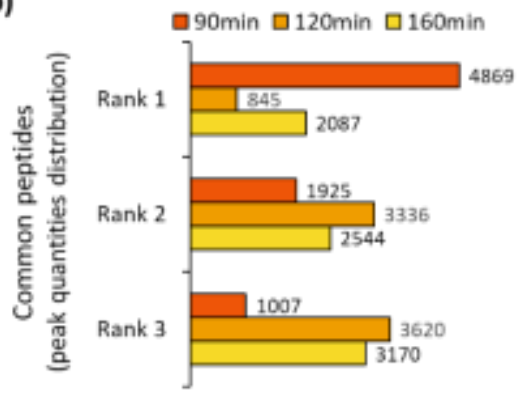

(c)

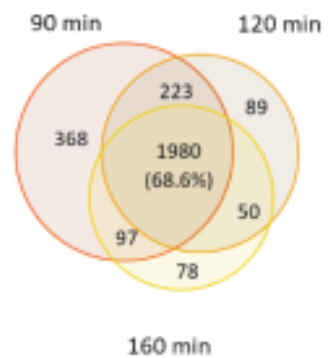

(d)

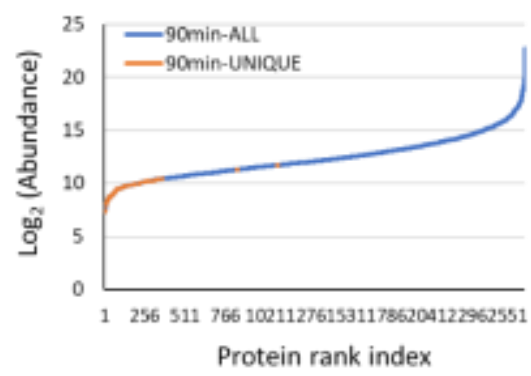

Figure S1. Optimization of different liquid chromatography gradient lengths in DIA-MS. (a) The number of protein groups, unique peptides and precursors identified from 1.5ng ( 10 cell equivalent) of PC-9 digests through different gradient lengths of liquid chromatography. (b) Comparison of ranking of peptides based on peak area abundance in commonly identified peptides. (c) Overlap of total proteins identified from different gradient lengths. (d) Protein abundance distribution of 368 uniquely identified proteins among total identified proteins in 90min gradient. 


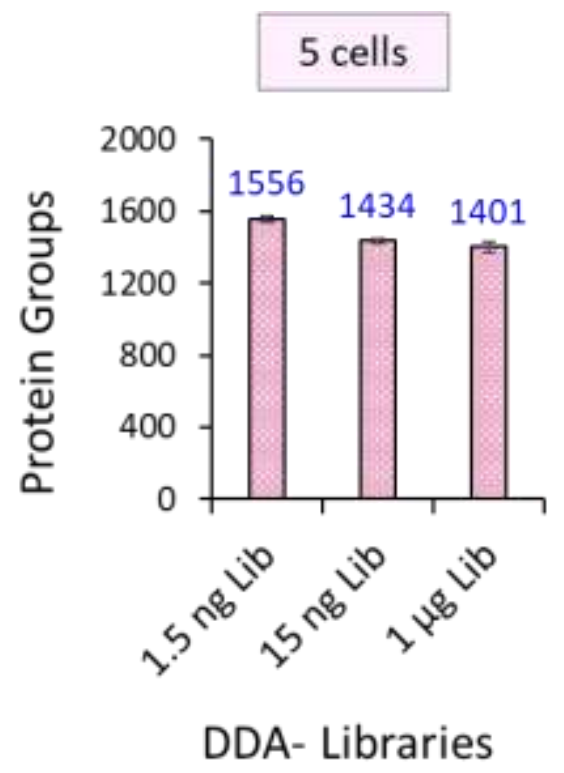

Figure S2. Effect of different sizes of DDA-specific spectra libraries on the performance of protein identification in $\mathbf{5}$ cells. Summary of the number of protein identification from 5 cells of PC-9 cells identified using small-size, medium-size and large-size libraries. 

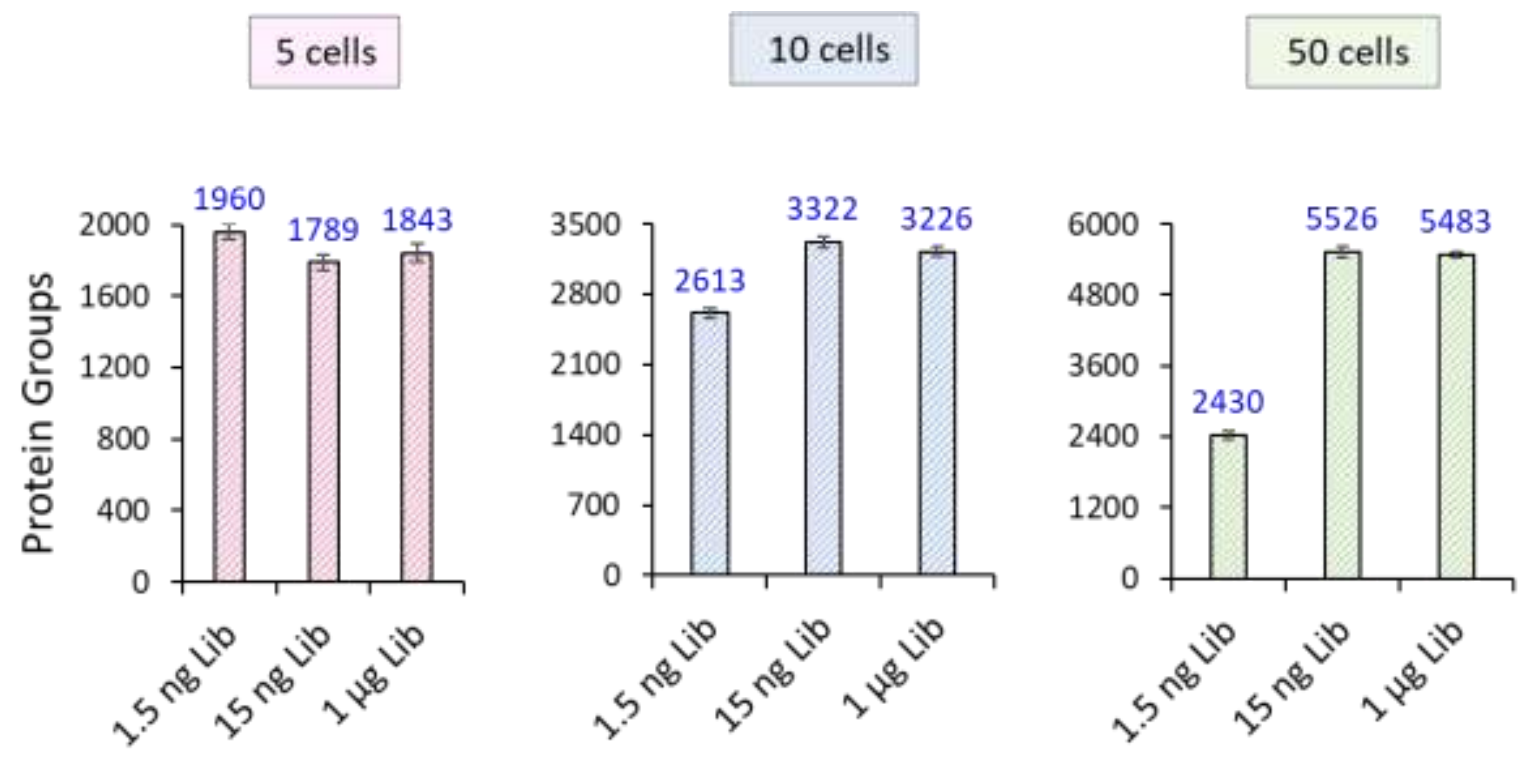

Libraries

Figure S3. Effect of different sizes of spectra libraries on the performance of protein identification in low-input cells by library-based DIA using DIA-NN. Summary on the number of protein identification from 5 cells, 10 cells and 50 cells of PC- 9 cells identified using small-size, medium-size and large-size libraries. 
(a)

$$
5 \text { cells }
$$$$
10 \text { cells }
$$

50 cells

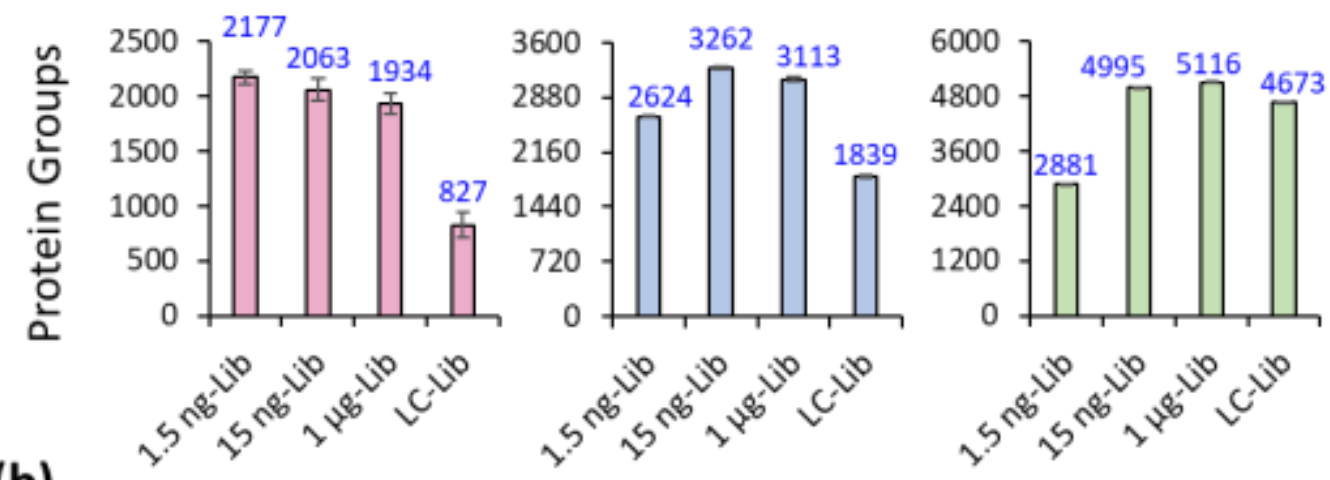

(b)

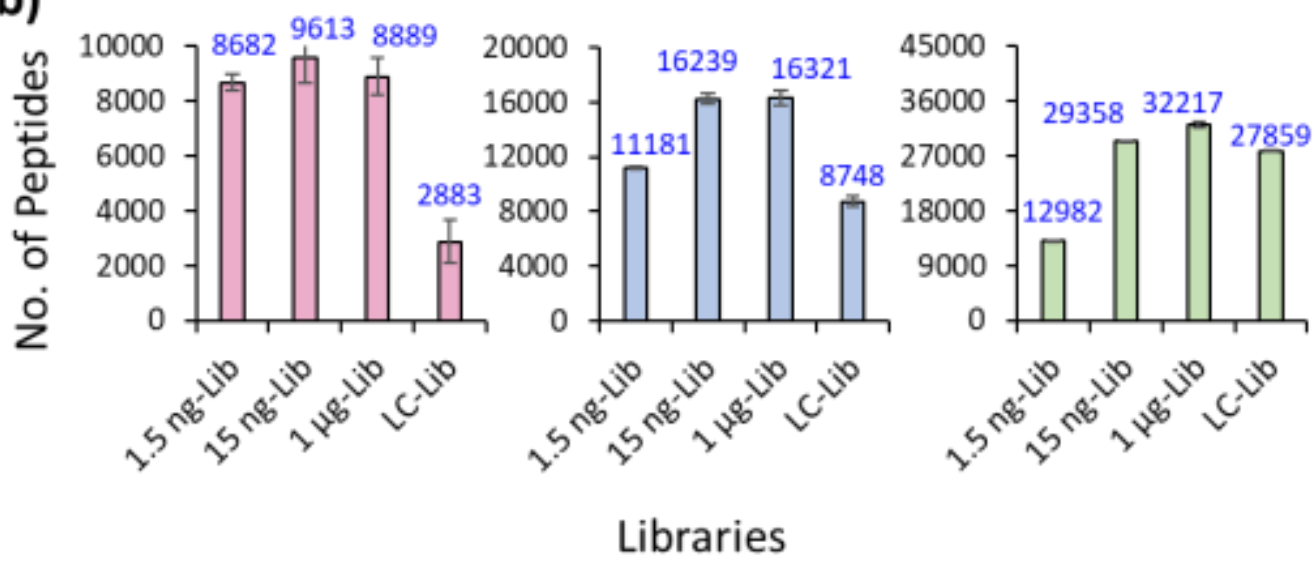

Figure S4. Effect of different sizes of spectra libraries on the performance of protein and peptide identification in low-input cells by library-based DIA obtained by Orbitrap Lumos mass spectrometer using Spectronaut. Influence of spectral libraries on the (a) number of protein identification and (b) unique peptides identified from 5 cells, 10 cells and 50 cells of PC- 9 cells obtained from small-size, medium-size and large-size libraries. 

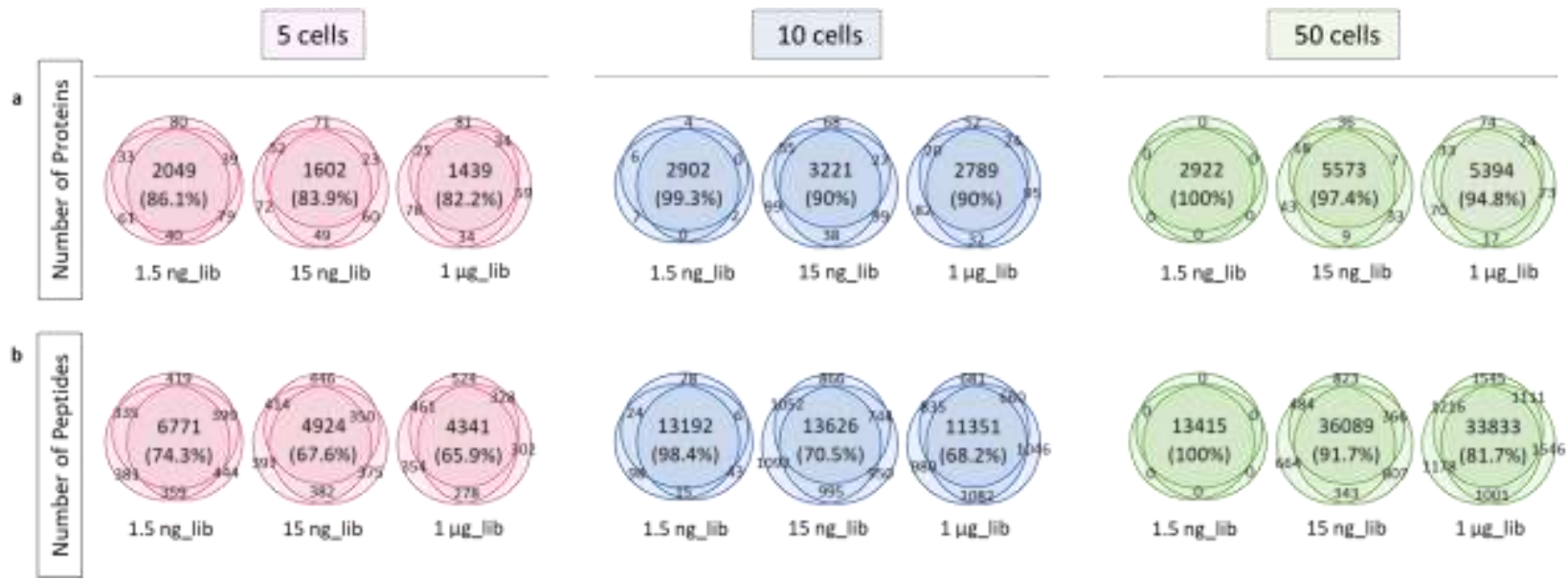

Figure S5. Evaluation of reproducibility in triplicate DIA-MS analysis identified by sample sizecomparable spectral libraries. (a) The overlaps of total identification of proteins and (b) unique peptides identified from triplicate DIA-MS runs for 5 cells, 10 cells and 50 cells of PC-9 digests as obtained from small-size, medium-size and large-size spectra libraries. 


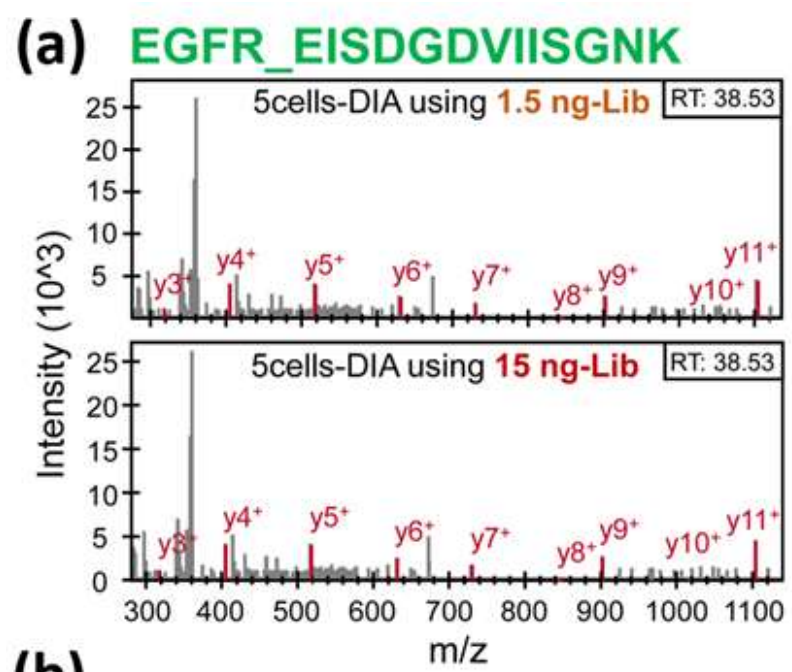

(b) $\mathrm{m} / \mathrm{z}$
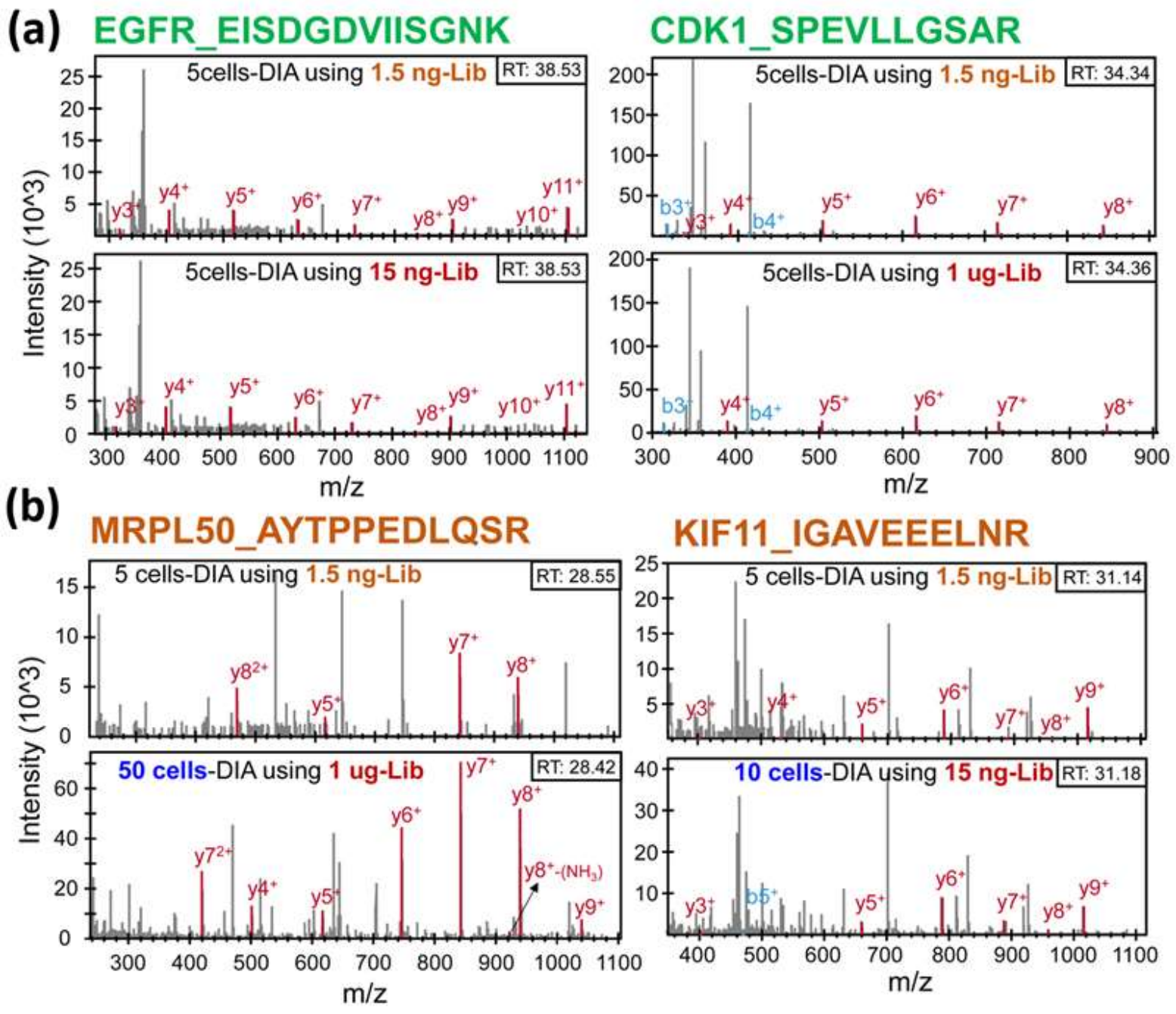

Figure S6. Evaluation on the spectral similarities of commonly and uniquely identified proteins in different libraries. (a) Fragment peak assignments of DIA-MS2 spectra of EGFR and CDK1 peptides using different libraries. (b) Fragment peak assignments of DIA-MS2 spectra of KIF11 and MRPL50 peptides using different libraries. 
(a)

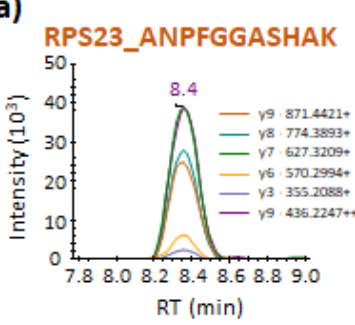

(b)
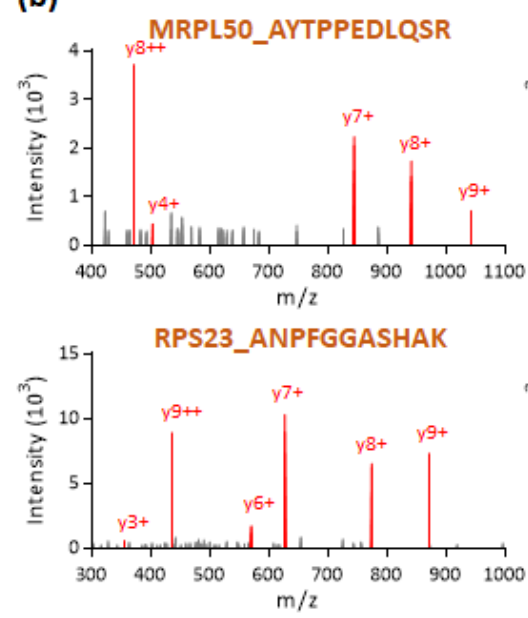
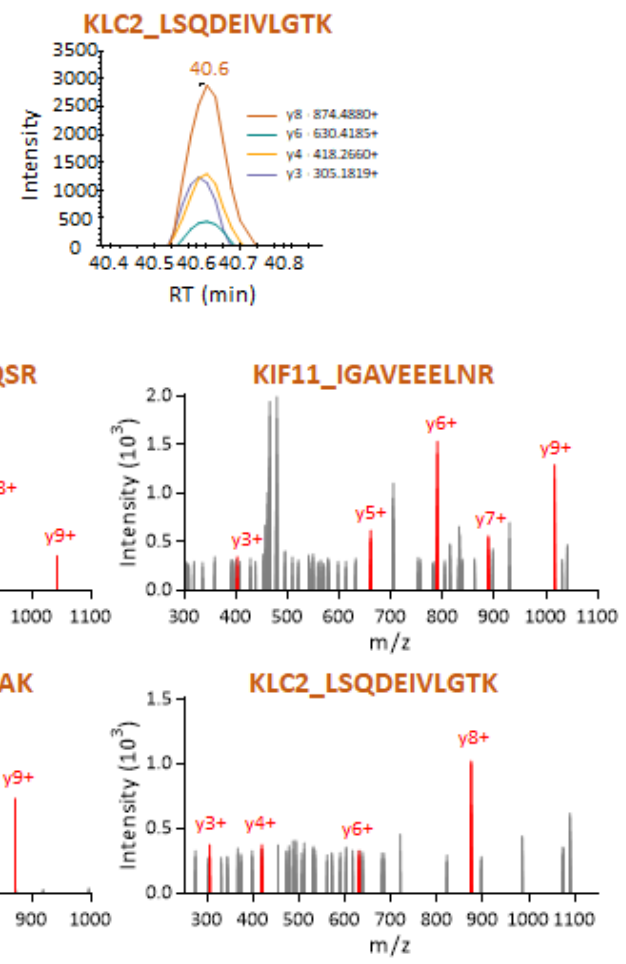

(c)
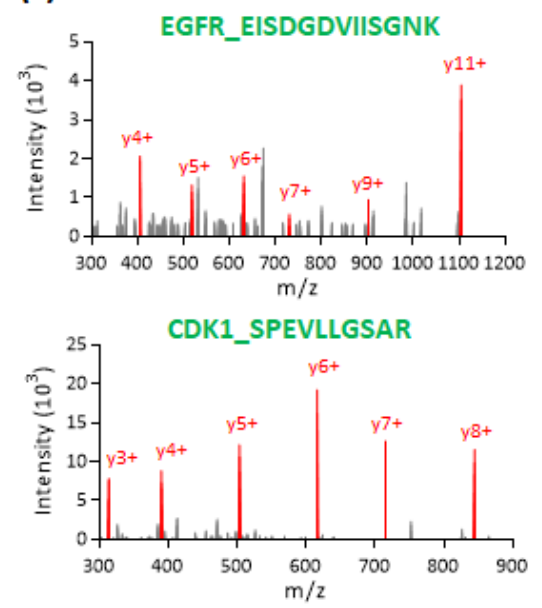

Figure S7. Validation of selected peptides identified in the 5-cells of PC-9 digests by parallel reaction monitoring (PRM) MS. (a) Extracted ion chromatograms (XICs) for additional two representative uniquely identified peptides mapped by the small-size library. PRM-MS2 spectra for the 4 uniquely identified peptides (b) mapped by the small-size library; and the 2 commonly identified peptides (c) mapped by the small-, medium-, and large-size libraries. 
Table S1. Detailed Parameters of DIA-MS2 optimization for low-input cells.

\begin{tabular}{|c|c|c|c|c|c|c|c|c|c|c|c|c|c|c|}
\hline \multirow{2}{*}{$\begin{array}{l}\text { MS2 } \\
\text { Resol. }\end{array}$} & \multirow{2}{*}{ Amount } & \multirow{2}{*}{ IW } & \multicolumn{3}{|c|}{$\begin{array}{l}\text { Identification results } \\
\text { (Total) }\end{array}$} & \multicolumn{3}{|c|}{$\begin{array}{l}\text { Identification results } \\
(\mathrm{CV}<20 \%)\end{array}$} & \multicolumn{2}{|c|}{$\begin{array}{c}\text { Median data } \\
\text { points per peak }\end{array}$} & \multicolumn{2}{|c|}{ Cycle Time } & \multicolumn{2}{|c|}{$\%$ of MS scans } \\
\hline & & & PG & PEP & Precursors & PG & PEP & Precursors & MS1 & MS2 & MS1 & MS2 & MS1 & MS2 \\
\hline \multirow{4}{*}{$30 K$} & $1500 \mathrm{pg}$ & 10 & 2044 & 8214 & 8484 & 1594 & 5426 & 5546 & 4 & 4 & 3.205 & 3.205 & $46.2 \%$ [50ms] & $100 \%$ [54ms] \\
\hline & $1500 p g$ & 15 & 1767 & 7067 & 7302 & 1420 & 4854 & 4953 & 5 & 5 & $2.30 \mathrm{~s}$ & $2.30 \mathrm{~s}$ & $41.7 \%$ [50ms] & $100 \%[54 \mathrm{~ms}]$ \\
\hline & 1500pg & 20 & 1655 & 5846 & 6042 & 1335 & 3906 & 4009 & 6 & 6 & $1.82 \mathrm{~s}$ & $1.82 \mathrm{~s}$ & $54 \%$ [50ms] & $99.9 \%$ [5.4ms] \\
\hline & $1500 \mathrm{pg}$ & 25 & 1699 & 6101 & 6287 & 1325 & 3936 & 4011 & 8 & 8 & $1.55 \mathrm{~s}$ & $1.55 \mathrm{~s}$ & $65.6 \%$ [50ms] & $99.9 \%$ [54ms] \\
\hline \multirow{4}{*}{$60 \mathrm{~K}$} & $1500 \mathrm{pg}$ & 10 & 3051 & 13767 & 14348 & 2560 & 10223 & 10522 & 3 & 3 & 5.825 & 5.825 & $63.6 \%$ [50ms] & $99.8 \%$ [118ms] \\
\hline & 1500pg & 15 & 2932 & 12992 & 13524 & 2364 & 8589 & 8831 & 3 & 3 & $4.09 \mathrm{~s}$ & $4.09 \mathrm{~s}$ & $52.1 \%$ [50ms] & $99.7 \%$ [118ms] \\
\hline & $1500 \mathrm{pg}$ & 20 & 2821 & 11285 & 11703 & 2436 & 8554 & 8795 & 4 & 4 & $3.17 \mathrm{~s}$ & $3.17 \mathrm{~s}$ & $73.1 \%[50 \mathrm{~ms}]$ & $99.3 \%[118 \mathrm{~ms}]$ \\
\hline & $1500 \mathrm{pg}$ & 25 & 2530 & 9797 & 10148 & 2105 & 7303 & 7417 & 5 & 5 & 2.645 & 2.645 & $52 \%[50 \mathrm{~ms}]$ & $99.1 \%$ [118ms] \\
\hline \multirow{4}{*}{$120 \mathrm{~K}$} & $1500 \mathrm{pg}$ & 10 & 2838 & 12187 & 12632 & 1663 & 5234 & 5294 & 2 & 2 & $11.04 \mathrm{~s}$ & $11.04 \mathrm{~s}$ & $21.5 \%$ [50ms] & $98.9 \%$ [246ms] \\
\hline & $1500 \mathrm{pg}$ & 15 & 3014 & 13623 & 14201 & 2416 & 8861 & 9011 & 2 & 2 & 7.665 & 7.665 & $55.4 \%[50 \mathrm{~ms}]$ & $97.6 \%[246 \mathrm{~ms}]$ \\
\hline & 1500pg & 20 & 3004 & 12228 & 12658 & 2585 & 9329 & 9473 & 3 & 3 & $5.84 \mathrm{~s}$ & $5.84 \mathrm{~s}$ & $19.4 \%$ [50ms] & $79.8 \%$ [246ms] \\
\hline & $1500 \mathrm{pg}$ & 25 & 2890 & 12003 & 12458 & 2543 & 9579 & 9773 & 3 & 3 & $4.79 \mathrm{~s}$ & $4.79 \mathrm{~s}$ & $40.7 \%$ [50ms] & $78.7 \%$ [246ms] \\
\hline
\end{tabular}

MS2 Resol. = MS2 Resolution

IW = Isolation Window

$P G=$ Protein Groups

PEP $=$ Peptides 
Table S2. Summary of proteome coverages of the three DIA-specific spectral libraries.

\begin{tabular}{|c|c|c|c|c|}
\hline \multicolumn{5}{|c|}{ DIA-based libraries - Library size / depth } \\
\hline & Library & Protein Groups & Peptides & Precursors \\
\hline 1 & PC9-1500pg-DIAlib (Small) & 2924 & 13415 & 14078 \\
\hline 2 & PC9-15ng-DIAlib (Medium) & 5848 & 40562 & 47205 \\
\hline 3 & PC9-1ug-DIAlib (Large) & 6452 & 53297 & 65894 \\
\hline
\end{tabular}


Table S3. Summary of proteome coverages of the three DDA-specific spectral libraries.

\begin{tabular}{|c|c|c|c|c|}
\hline \multicolumn{5}{|c|}{ DDA-based libraries - Library size / depth } \\
\hline & Library & Protein Groups & Peptides & Precursors \\
\hline 1 & PC9-1500pg-DDAlib (S) & 2377 & 8880 & 9508 \\
\hline 2 & PC9-15ng-DDAlib (M) & 4558 & 22159 & 26004 \\
\hline 3 & PC9-1 & 6234 & 44182 & 58443 \\
\hline
\end{tabular}

